\title{
A PROPOS DE L'UNICITÉ DE SOLUTION DANS LES PROBLĖMES DE RÉSEAUX MAILLÉS
}

ANGLÈS D'AURIAC

Ancien élève de I'École Polytechnique

Tn probleme de réseaux maillés se laisse en seneral schématiser de la facon suivante:

On a un ensemble de points appelés nouds on sommets et un ensemble de lignes appelés cotés dont chacun réunit un noeud it un noeud.

Chaque coté, qui représentesune conduite, est caractérisé par une certaine loi de perte de charge en fonction du débit $\Delta \mathrm{H}=f(\mathrm{Q})$.

Un certain nombre de sommets possèdent une communication avec l'extérieur ; c'est-à-dire que, par eux, du débit peut entrer dans le réseau, ou en sortir. Ces sommets sont apperés issues.

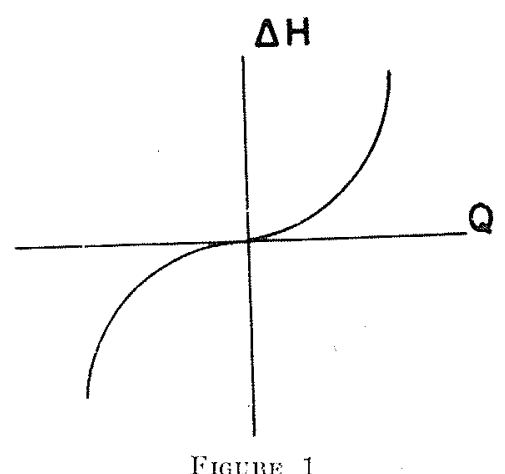

On se donne aux issues un certain nombre de conditions soit de pression, soit de débit et l'on cherche s'il existe un écoulement et un senl compatible avec ces conditions.

Nous allons démontrer que, quand il y a une solution, elle est toujours unque à la seule condition que toutes les caractéristiques des conduites soient croissantes de - $-\infty$ à $+\infty$.

\section{CONDITIONS DE DEBIT}

Supposons que tous les débits aux issues soient donnés (il faut évidemment que leur somme fasse zéro) et supposons qu'il existe un certain écoulement $\mathrm{E}_{\mathrm{i}}$ compatible avec ces données. Nous aurons en chaque noud $\Sigma Q=0$ en affectant par exemple du signe + les débits allant vers le noend et du signe - les débits renant du noud. Nous aurons également poux toul contour fermé tracé dans le réseau $\Sigma \Delta H=O$.

Nous voulons démontrer qu'il ne peut exister un autre ceoulement $\mathrm{E}_{2}$ satisfaisant à ces conditions et compatible avec les débits donnés aux issues.

Supposons en efret que $\mathrm{E}$, existe. Considérons l'écoulement fictif $\mathrm{E}^{\prime}=\mathrm{E}_{1}-\mathrm{E}_{\text {, c'est-à- }}$ dire tel que dans chaque conduite le débit soit la différence entre le débit dans l'écoulement $E_{\text {, }}$ et le débit dans l'écoulement $\mathbf{E}_{2}$. Les éconlements $E_{1}$ et $E_{2}$ sont par hypothèse des écoulements réels satisfaisant à toutes les conditions de débit ei de pression tandis que l'écoulement $E^{\prime}$ ne satisfait, par sa définition même, qu'aux conditions de débit, c'est pourcuoi nous l'appelons écoulement fictif. Pour le réaliser, il faudrait introduire dans le réseau tout un système de pompes et de résistances.

L'écoulement $\mathrm{E}$ ' est un écoulement fermé c'est-à-dire que ie débit aux issues est nul. Or tout écoulement fermé jouit de la propriété suivante: on peut toujours trouver un certain circuit fermé parcouru dans le même sens.

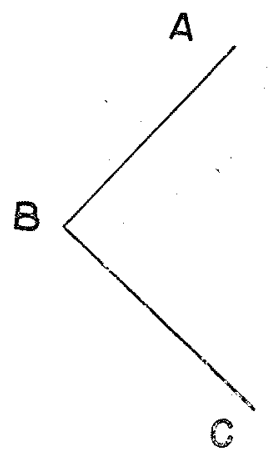

FIGURE 2

Partons en effet d'un còté $A B$ pris au hasard parcouru de $A$ en $B$. Du noud $B$ partira au moins une conduite telle que $B C$ parcouru de $B$ en $C$. Nous pouvons done former une ligne de plus en plus longue et parcourue dans le 
mème sens. Il faut bien que cette ligne se recoupe à un moment donné, ce qui démontre l'existence d'un circuit fermé parcouru dans lo mème sens (nous ne disons pas bien entendu avec le mème débit).

Soit $y$ un tel circuit fermé. Prenons comme sens positif des débits dans $y$, le sens de parcours dans l'écoulement E'.

Le long du contour $y$ mous avons, dans l'écoulement $\mathrm{E}_{1}$ :

$$
\Sigma \Delta H==0
$$

En passant de $\mathrm{E}_{1}$ à $\mathrm{E}_{2}$, tous les $\mathbf{Q}$ sont augmentés, done tous les $\Delta \mathbf{H}$ le seront aussi puisque toutes les caractéristiques sont croissantes. Il est donc impossible d'avoir $\Sigma \Delta H=O$, le long de $y$ dans l'écoulement $\mathrm{E}_{2}$. L'écoulement $E_{: i}$ ne peut donc pas exister.

\section{CONDITIONS DE PRESSION}

Supposons maintenant qu'au lieu de conmaìtre les débits aux issues, nous connaissions les pressions. Supposons qu'il existe un écoulement réel $E_{1}$ compatible avec ces pressions aux issues et avec les caractéristiques des conduites. Nous disons qu'il ne peut exister un autre écoulement réel $\mathrm{E}_{2,}$.

Formons en effet l'écoulement E' défini de la facon suivante: la pression en chaque noud est la différence des pressions dans l'écoulement $\mathrm{E}_{1}$ et l'écoulement $\mathrm{E}_{2}$. L'écoulement $\mathrm{E}^{\prime}$ est un écoulement virtuel qui satisfait évidem. ment aux conditions de pression mais non de débit.

Dans l'écoulement E', les pressions sont nulles aux issues. Mais elles ne peuvent pas être nulles en tous les nœuds sinon $E_{1}$ et $E_{2}$ seraient identiques. Il $\mathrm{y}$ a donc au moins um noud qui dans $E^{\prime}$ a une pression positive ou négative.

Supposons-la par exemple positive, et cherchons le ou les nouds ayant la pression maximum. Il en existe au moins un. Soit A un tel

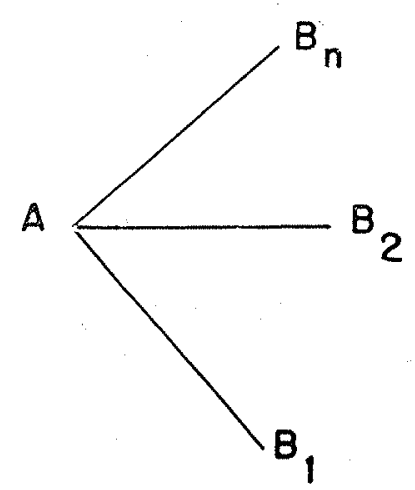

Figure 3 noeud et soil $H_{\mu}$ la pression y régnant (dans l'écoulement $\mathbf{E}^{\prime}$ ).

Tracons l'étoile des conduites autour de $A$, les pressions en $B_{1}, B_{2}, B_{n}$ sont inférieures ou au maximum égales à la pression $H_{m}$. Si elles sont toutes égales à $H_{m}$, recommençons à tracer l'étoile autour de $B_{1}$ par exemple. Ce faisant, nous élargissons de plus en plus la zone où règne la pression maximum. Mais cette zone ne peut s'étendre à tout le réseau car les pressions sont nulles aux issues.

Il y a donc forcément un certain noud $C$ oit règne la pression $H_{m}$ et duquel partent un certain nombre de conduites à pression constante $\mathrm{H}_{n}$ et d'autres conduites à pression décroissante de $\mathrm{H}_{m}$ a $h_{n}$. Placons-nous en un tel noeud.

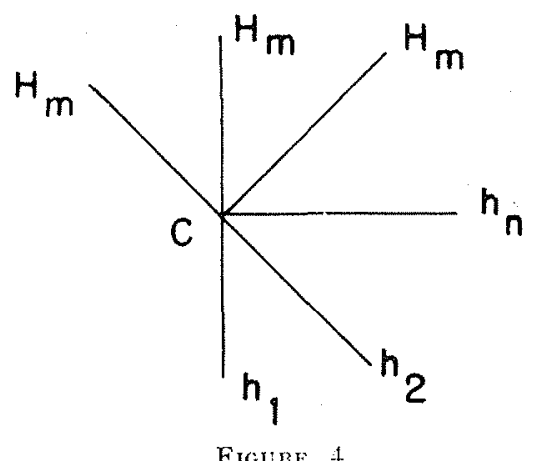

Dans l'écoulement $\mathrm{E}_{1}$, nous avons en ce noeud $\Sigma Q=O$. En passant de $E_{1} \dot{a} E_{2}$ certains $\Delta H$ sont inchangés, les autres varient tous dans le mème sens. Les $Q$ correspondanis varieront eux aussi dans le même sens. On ne peut donc pas avoir $\Sigma Q=O$ dans l'écoulement $E_{2}$ au noud $C$. L'écoulement $E_{2}$ ne peut donc exister.

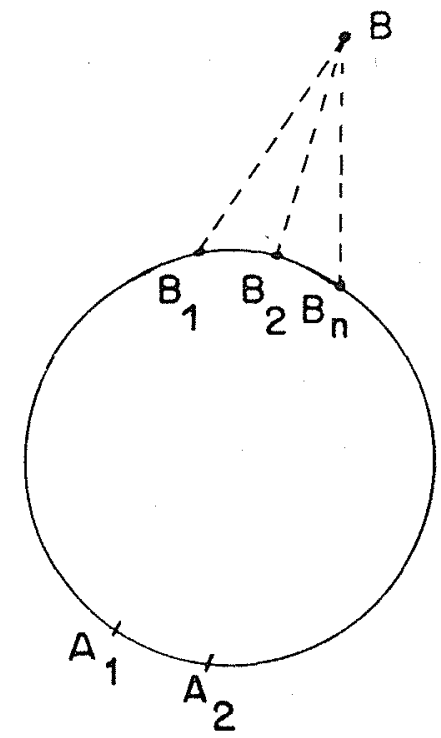

Figure 5 


\section{GAS GENERAL}

Considérons un réseau mailé pour lequel certaines issues $A_{1}, A_{2} \ldots$ aient un débit connu, et d'autres issues $B_{1}, B_{2} \ldots$ une pression connue. Nous voulons démontrer que si ane solution existe, elle est unique.

Rémissons par des conduites fictives, les points $B_{1}, B_{2} .$. , etc, à un point $B$ où règne une pression donnée $H$. Les conduites ficlives $B_{1} B$, $B_{2} B$, etc. ont des caractéristiques horizontales $\Delta \mathrm{H}=$ Cte. Le point $\mathrm{B}$ est une issue dont on connait le débit. Nous retombons donc sur le cas I.

Le raisonnement que nous avons fait dans te cas 1 ne serait plus valabie si toutes les conduites du contour $Y$ avaient les caractiristiques horizontales $\Delta \mathrm{H}=$ Cte. Or, le réseau des conduites fictives $B, B, B, B$, efc a la forme d'une étoile. C'est un réseau ramifié et non maillé. Il est impossible d'y tracer un contour lerme $\gamma$. Le contour $\gamma$ possede done au moins une conduile qui ne fait pas partie des conduites fictives et le raisommement que nous avons fail dams le cas 1 reste valable.

\section{CONCLUSION}

L'unicité de la solution dépend uniquement du lait que les caractéristiques des conduites sont des fonctions croissantes. II est inutile que les courbes passent par l'origine. Nous pouvons. donc intercaler dans le réseau on aux issues ur nombre quelconque de pompes, de turbines, ou de résistances mème dissymétriques. Nous sommes assurés de l'unicité de la solution pourvu que les organes puissent être représentés par des conduites fictives à caractéristique croissante.

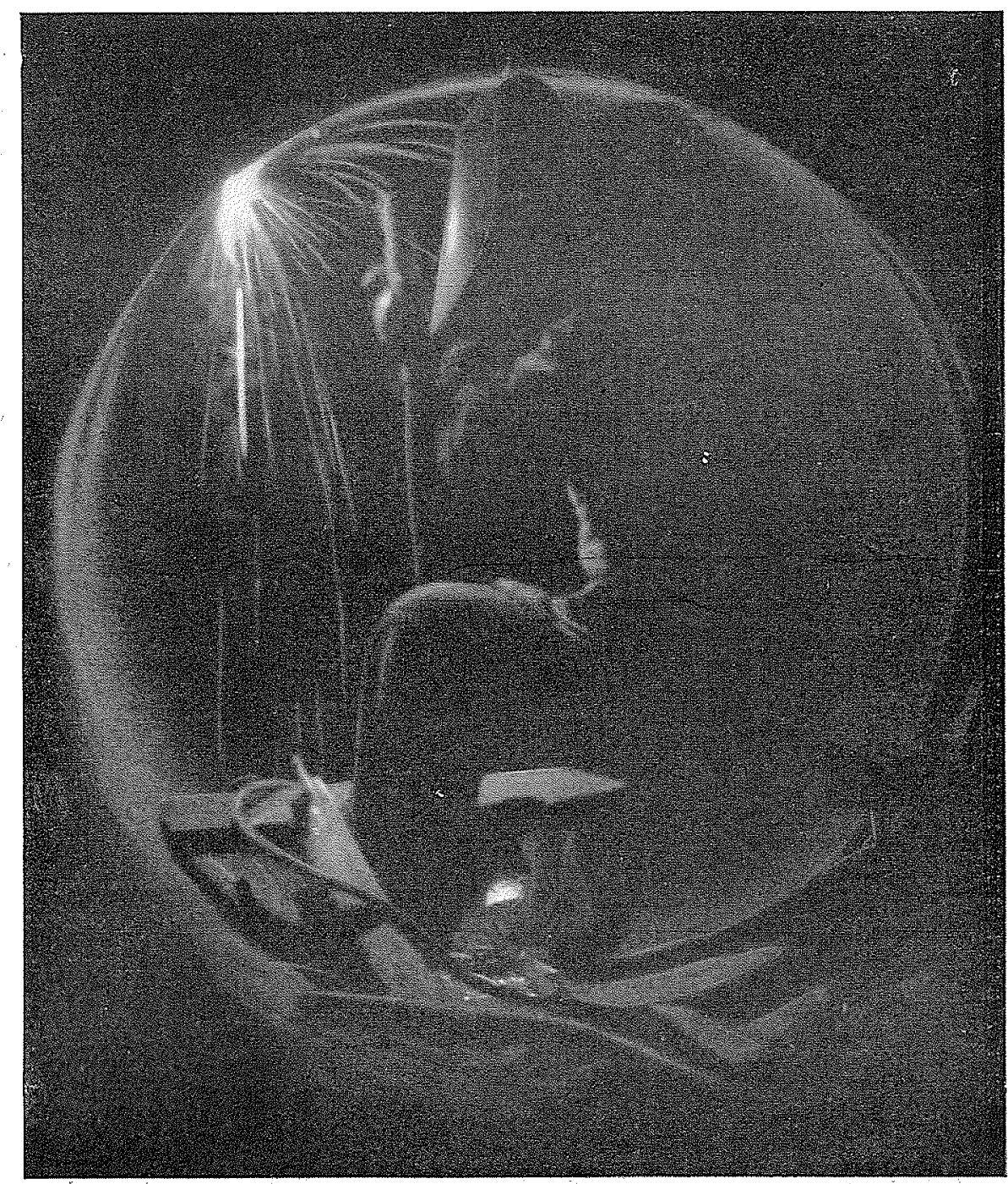




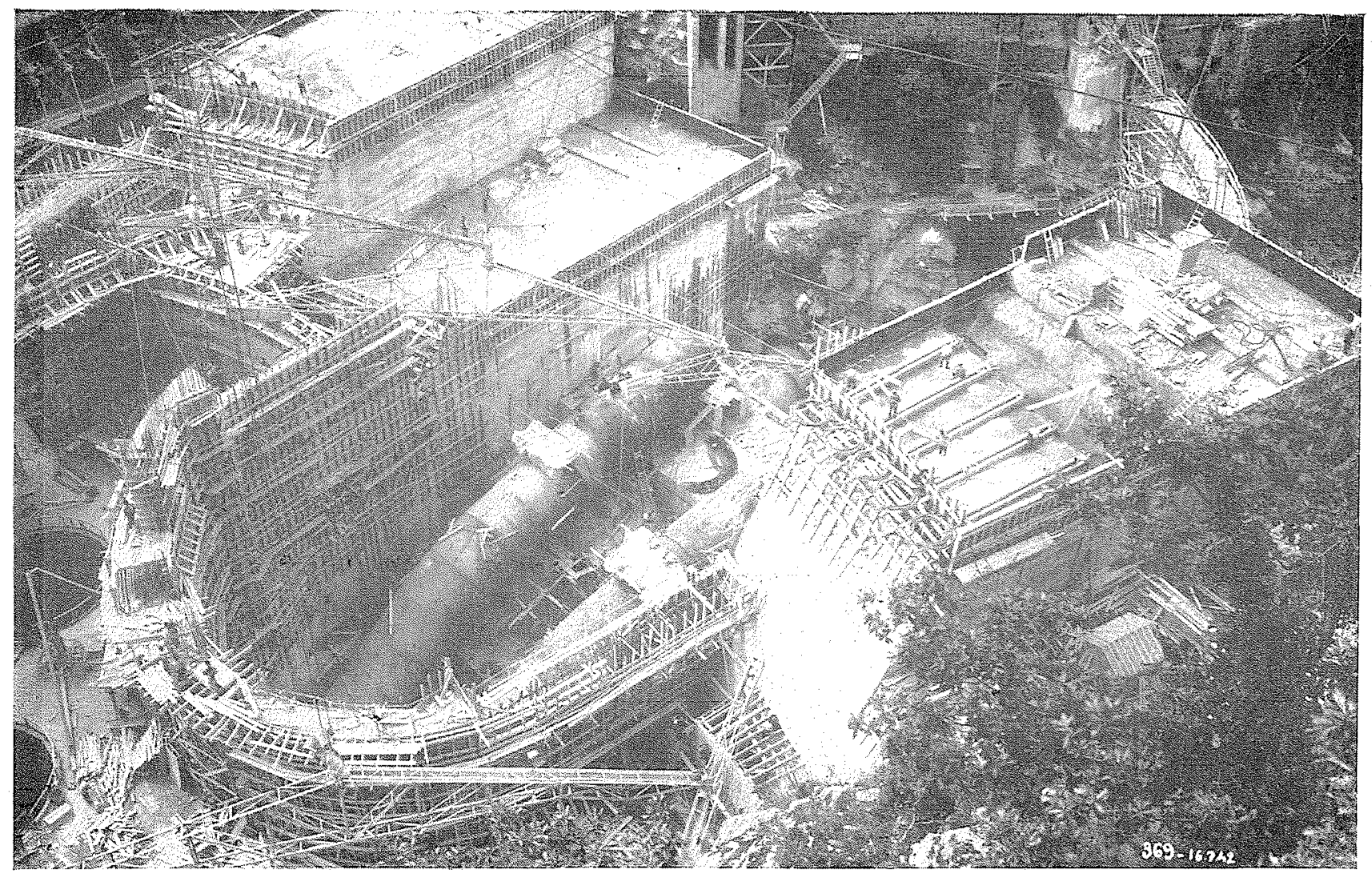

INSTALATON DES CONDELTES FORCSES DE BABRAGE DE LAYGE

(Cliché Escher Wu:

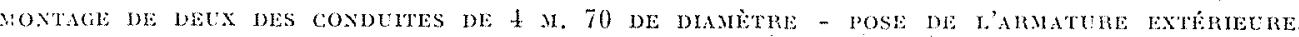

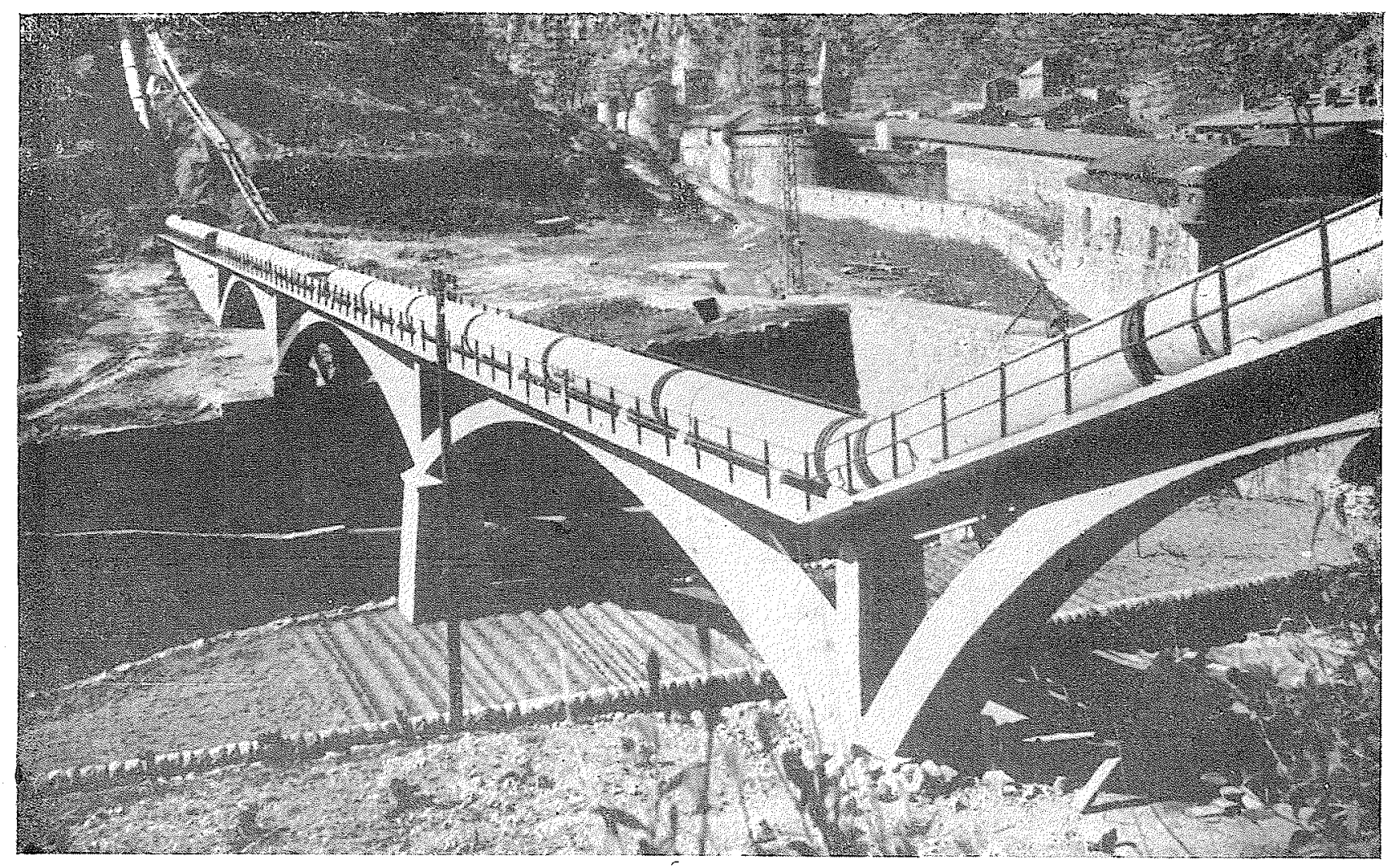

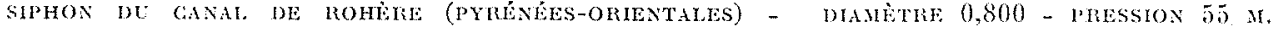

